\title{
Fringe jump analysis and implementation of polarimetry on the ASDEX Upgrade DCN interferometer ${ }^{\mathrm{a})}$
}

\author{
A. Mlynek ${ }^{1}$, b) L. Casali ${ }^{1}$, O.Ford ${ }^{2}$, H. Eixenberger ${ }^{1}$, and the ASDEX Upgrade Team ${ }^{\text {c) }}$ \\ ${ }^{1}$ Max-Planck-Institute for Plasma Physics, Garching 85748, Germany \\ ${ }^{2}$ Max-Planck-Institute for Plasma Physics, Greifswald 17491, Germany
}

\begin{abstract}
The ASDEX Upgrade tokamak is equipped with a 5-channel DCN interferometer with a probing wavelength of $195 \mu \mathrm{m}$. Up to now, phase measurement and density calculation have been accomplished by hard-wired phase counting electronics. Meanwhile, a fast digitizer has been installed which acquires the raw signals. That way, the various causes of counting errors by integer multiples of $2 \pi$, so-called fringe jumps, can be analyzed, and phase reconstruction schemes based on digital signal processing can be developed. In addition, a prototype polarimeter setup has been installed on one channel and allows for measurement of the Faraday rotation experienced by the probing beam.
\end{abstract}

\section{INTRODUCTION}

Interferometry is a standard method of measuring the electron density in a fusion plasma. The ASDEX Upgrade tokamak is equipped with both, a $10.6 \mu \mathrm{m} / 633 \mathrm{~nm}$ two-color interferometer, and a $195 \mu \mathrm{m}$ DCN interferometer. This article will focus on the DCN system, whose hard-wired readout electronics is currently being replaced by a digital signal processing approach in order to reduce the number of fringe jumps. For this purpose, a fast digitizer has recently been installed which acquires the detector raw signals. Based on this new data, the various causes of fringe jumps have been analyzed, as will be illustrated in section II of this article. Strategies to handle the corresponding signal perturbations will be discussed. In addition, polarimetry is currently being implemented, which delivers additional constraints for current profile reconstruction and provides a reference for the correction of remaining fringe jumps. Section III presents the first results obtained with the single-channel prototype polarimeter on ASDEX Upgrade. Section IV summarizes the results and gives an outlook on next steps.

\section{RAW SIGNAL ANALYSIS DURING FRINGE JUMPS}

The 5-channel DCN interferometer uses $195 \mu \mathrm{m}$ radiation from a DCN gas laser and employs heterodyne detection with a beat frequency of $10 \mathrm{kHz}$. A detailed description of the system can be found in Ref. ${ }^{1}$. The readout electronics measures the phase shift between the 10 $\mathrm{kHz}$ probe signal and a $10 \mathrm{kHz}$ reference signal, counting also integer multiples of $2 \pi$. Two different generations of readout electronics are currently operated in parallel, whose principle of operation is described in Ref. ${ }^{2}$ and Ref. ${ }^{1}$, respectively. Both of them show fringe jumps under certain plasma conditions. To investigate their cause,

\footnotetext{
a) Contributed paper published as part of the Proceedings of the 20th Topical Conference on High-Temperature Plasma Diagnostics, Atlanta, Georgia, June 2014.

b) Author to whom correspondence should be addressed: amlynek@ipp.mpg.de

${ }^{c)}$ For authors' list, see U. Stroth et al., Nucl. Fusion 53, 104003 (Year: 2013).
}

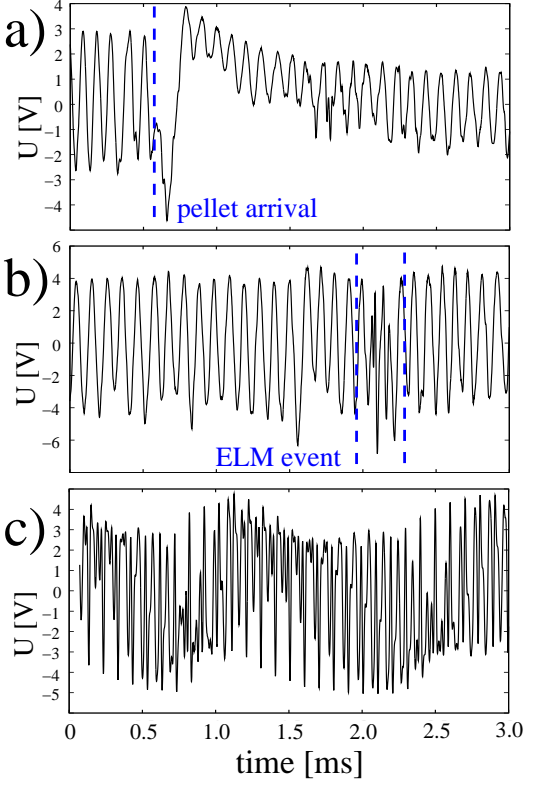

FIG. 1. Detector raw signals for different plasma scenarios: a) Injection of a pellet. b) During an ELM event. c) In presence of a neoclassical tearing mode. The line-integrated electron density for the three cases shown is roughly $6.0,5.0$ and $6.5 \times 10^{19} \mathrm{~m}^{-2}$, respectively.

the detector raw signals for all 5 channels, as well as the common reference signal, are now routinely digitized by a new data acquisition system with a sampling rate of 1 MSample/s, yielding an oversampling factor of 100 . That way, the characteristic signature of signal perturbations caused by beam refraction in the plasma can be analyzed. In the absence of perturbations, the signals are almost perfectly sinusoidal. Some typical plasma events which perturb the signal are shown in figure 1 and shall be discussed in the following.

\section{A. Pellet injection}

The injection of cryogenic deuterium pellets into the plasma results in a rapid increase of density and thus a rapid phase change, which is difficult to track. Without pellets, phase changes are usually much smaller than $2 \pi$ within the period of the $10 \mathrm{kHz}$ beat signal of $100 \mathrm{~ms}$, and can therefore be successfully followed. For small and medium-size pellets, this is still feasible. In case of large 
pellets, however, the phase change can be larger than $2 \pi$, and the situation becomes even more difficult due to the fact that pellets in addition cause a quick drop of the signal amplitude, which is strongest on edge channels and caused by beam refraction, as illustrated in figure 1a). On ASDEX Upgrade, the originally unipolar detector signal is shifted by a high-pass filter in the signal pre-amplifier to be bipolar and symmetric with respect to the zero line. Therefore, the sudden amplitude change results in a temporary DC offset of the signal, which settles back on the characteristic time scale of the high-pass filter $(\tau=1 \mathrm{~ms})$. Large pellets are therefore very challenging and even by digitally processing the acquired raw signal, an unambiguous phase reconstruction will probably not be achievable. Accordingly, additional information will be required for proper density reconstruction, such as the Faraday angle measured by polarimetry.

\section{B. Edge-localized modes (ELMs)}

The occurrence of ELMs in an H-mode discharge mainly affects the lateral interferometer channels. The ELM crash results in a sudden decrease of density and accordingly in a rapid phase change in the detected signal, which can be of the order of $2 \pi$. Subsequently, density returns to its original value, until the next ELM crash occurs. Also here, the challenge therefore is to track fast phase changes. This can get more difficult when filamentary structures of high density (ELM filaments) appear in the scrape-off layer and eventually cross the line of sight of the interferometer. In many ELM phases, short dips in the signal are observed, which are supposed to be caused by beam refraction on high-density filaments. Figure 1b) shows an example, where a fast up and down of the signal level around $t=2 \mathrm{~ms}$ can be seen, although density in this time window was found not to vary much. Sudden phase changes due to ELM crashes and short signal distortions due to filaments therefore need to be distinguished, which might sometimes be ambiguous. In such cases, the a priori knowledge that the inter-ELM density tends to vary only slowly, or to be even constant in H-mode flat-top phases, is helpful to make the proper fringe correction, once a perturbation of the signal has been identified as ELM-related.

\section{Core Magneto-hydrodynamic (MHD) modes}

A variety of MHD modes, such as neoclassical tearing modes (NTMs), might be present in the core and midradius part of the plasma. The magnetic island(s) related to a mode tend to have a local density that differs from the surrounding plasma. Accordingly, there is a density gradient on the boundary of the island, which periodically crosses the line of sight of the interferometer in case of a rotating mode. That way, the DCN laser beam periodically suffers from refraction and gets either de-focused or laterally deflected, resulting in a periodic drop of the amount of laser power arriving at the detector. Accordingly, the $f_{0}=10 \mathrm{kHz}$ beat signal experiences amplitude modulation at the mode frequency $f_{M H D}$, resulting in a detector voltage

$$
U(t)=U_{0} \sin \left(2 \pi f_{0} t\right) \cdot\left[U_{0}+U_{1} \sin \left(2 \pi f_{M H D} t\right)\right]
$$

where $U_{1}<U_{0}$ is the modulation amplitude. This can equivalently be written as
$U(t)=U_{0} \sin \left(2 \pi f_{0} t\right)-U_{1} / 2 \cos \left(2 \pi\left(f_{0}+f_{M H D}\right) t\right)+$

$+U_{1} / 2 \cos \left(2 \pi\left(f_{0}-f_{M H D}\right) t\right)$

Since the cosine is an even function, this means that two additional frequency components $f_{0}+f_{M H D}$ and $\left|f_{0^{-}} f_{M H D}\right|$ besides $f_{0}$ appear in the frequency spectrum. Figure 1c) shows an example time trace of the detector signal in presence of MHD activity. In addition, figure 2 presents a spectrogram of the signal for a longer time window, illustrating the additional frequency components that occur due to MHD activity. That way, the interferometer can act as MHD detector, which provides some spatial resolution due to its different lines of sight, allowing for crude mode localization. The extraction of $f_{M H D}$ from the spectra, however, needs to take into account that each MHD frequency appears twice in the spectrogram. The signal distortion due to MHD activity

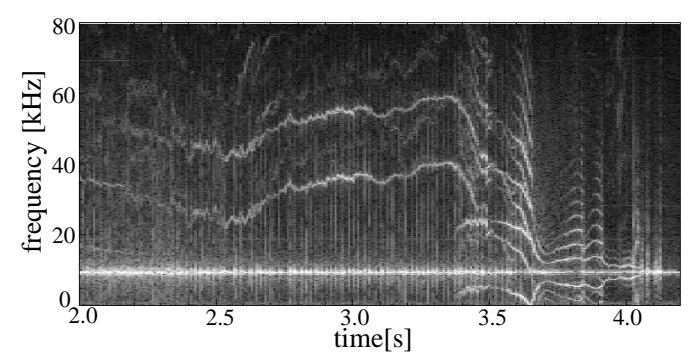

FIG. 2. Spectrogram of the detector signal for an inner interferometer channel in the presence of neoclassical tearing modes (NTMs, in ASDEX Upgrade discharge \#29487). Figure 1c) corresponds to $t=3.63 \mathrm{~s}$ in this discharge.

can perturb proper phase and density calculation and therefore needs to be removed by digital band-pass filtering of the signals, to allow only the $f_{0}$ component to pass through. The closer an MHD-related frequency component is to $f_{0}$, the narrower the filter pass-band has to be. If $f_{0}$ is crossed by an MHD component of large amplitude, phase reconstruction may temporarily become impossible, resulting in unavoidable fringe loss. To sum up, some events in the plasma can heavily perturb the interferometer signal, with each one having its very characteristic signature. Band-pass filtering the detector signals can remove the perturbation in some cases, like for core MHD modes, but also restricts the ability of the interferometer to follow fast density changes, and is therefore counterproductive in case of pellet injection or ELM crashes. Accordingly, when performing density reconstruction by digital processing of the raw signals, identification of the kind of perturbation and appropriate selection of the filter bandwidth will be of high importance.

\section{POLARIMETRY}

On ASDEX Upgrade, which used to have a pure DCN interferometer, a prototype polarimeter setup has been recently installed on one of the five interferometer channels. It measures the Faraday rotation of the probing 
beam, which is proportional to the line integral of $n_{e} \cdot B_{\|}$, electron density times magnetic field component in beam direction. Two wire grid polarizers have been added to define the polarization of the probing beam entering the plasma, and of the frequency-shifted beam used for beat signal generation. After plasma crossing and beam combination, a third wire grid was installed which acts as polarizing beam splitter, separating ordinary and extraordinary components, which are then measured by individual detectors. The detector signals are both digitized at $1 \mathrm{MSample} / \mathrm{s}$, and their amplitude ratio and their relative phase is calculated by digital signal processing. The Faraday angle is given by the arc tangent of the amplitude ratio of the two detector signals, and the deviation of their relative phase from zero gives the amount of ellipticity generated by the Cotton-Mouton-effect. Since the two detectors may have different sensitivity, the polarimeter requires calibration, which delivers an additional calibration constant in the argument of the arc tangent. For calibration, ideally a linearly polarized beam should be used, whose angle of polarization continuously rotates, while the intensity remains constant. Attempts have been made to achieve this rotation of polarization with a $2.045 \mathrm{~mm}$ thick X-cut quartz plate, which should act like a half-wave plate for $195 \mu \mathrm{m}$ radiation according to the refractive indices for ordinary $\left(n_{o}=2.1116\right)$ and extraordinary $\left(n_{e o}=2.1592\right)$ beam found in literature ${ }^{3}$. However, this plate was found to generate, depending on orientation, a considerable amount of ellipticity, for still unknown reasons. For a linearly polarized beam, the phase shift between ordinary and extraordinary component should always be either $0^{\circ}$ or $180^{\circ}$, alternating whenever the intensity of one component reaches zero. But in the experiment, we observed that the intensity of the ordinary component varied by only about $30 \%$, i.e. never dropped to zero, and the phase shift varied from $0^{\circ}$ to $70^{\circ}$ and from $180^{\circ}$ to $250^{\circ}$, with a jump whenever the intensity of the extraordinary component dropped to zero. Therefore, the calibration setup was replaced by a quarter-wave plate, to make the beam circularly polarized, and a subsequent, rotating wire-grid polarizer. This new setup reliably generates a linearly polarized beam, just the intensity may vary during a turn in case of imperfections of the quarter-wave plate. The polarizer was rotated at constant angular velocity, and the calibration constant chosen in a way that the measured angle shows linear time dependence. Any intensity modulations cancel out, since only the amplitude ratio enters into the analysis.

Since the beginning of the 2014 experimental campaign on ASDEX Upgrade, Faraday rotation measurements have been carried out for some hundreds of plasma discharges. Figure 3 presents the time trace of the measured Faraday angle and the corresponding line-integrated density measured by the interferometer (scaled to overlap). Also, the temporal evolution of the plasma current and the plasma stored energy are shown. When the plasma current has reached its flat-top, some time elapses (see vertical dashed lines) until Faraday angle and density start being proportional. This reflects the current diffusion time. Towards the end of the discharge, the plasma suffers from core impurity accumulation and radiative

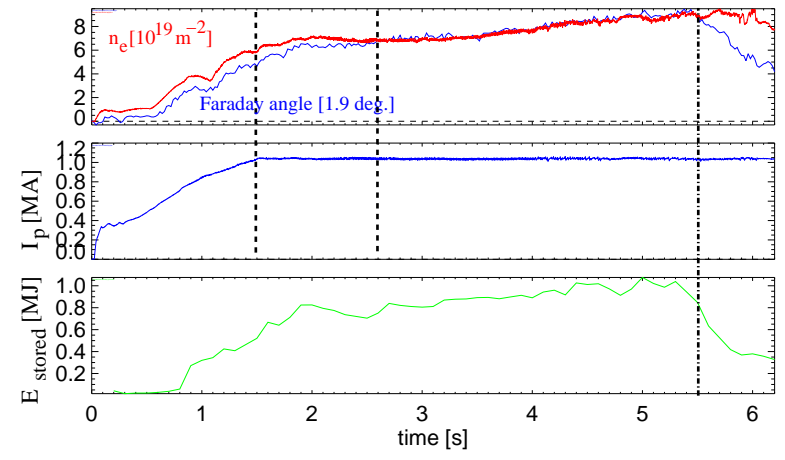

FIG. 3. Measured Faraday angle and line-integrated density (top) for an interferometer channel situated at mid-radius in ASDEX Upgrade discharge \#30507. In addition, the time trace of the plasma current (middle) and the stored energy (bottom) is shown.

cooling, visible as a drop of the stored energy. The dashdotted line roughly marks the onset time. The increased core resistivity causes broadening of the current profile and a drop of the Faraday angle measured by a probing chord at mid-radius. The noise level of the polarimeter is of the order of $+/-0.1$ degrees during flat-top phases and after the discharge. This value holds when averaging over time periods of $20 \mathrm{~ms}$. In transient phases, however, like during density ramps, fluctuations of up to 1 degree are regularly observed on the signal. This increased noise level in non-stationary phases is currently being analyzed, and possible countermeasures will be tested in the near future.

When analyzing the relative phase of the two detector signals, which allows for measuring the Cotton-Mouton effect, it was found that the phase shift is non-zero even when there is no plasma in the vessel. Typically, values of $20^{\circ}$ to $25^{\circ}$ are measured when the beam entering the vessel is close to ordinary polarization. This indicates that elliptical polarization is present even in the absence of the Cotton-Mouton effect. The optical properties of the vacuum windows, which are made from Z-cut quartz and should in theory preserve the polarization, therefore require detailed characterization, which is the subject of ongoing work. Obviously, the phenomenon of 'spurious ellipticity', which is described in literature for other DCN polarimeters $^{4}$, is currently also present on the ASDEX Upgrade system. It is our intention to identify the cause of spurious ellipticity and to test possible countermeasures.

\section{SUMMARY}

The detector raw signals of the DCN interferometer are now routinely digitized on ASDEX Upgrade, and different causes of fringe jumps have been identified and related to specific plasma conditions causing signal perturbation. A future digital signal processing algorithm for density reconstruction will have to include a handling 
strategy for each kind of perturbation. In addition, a polarimetric measurement has been recently implemented on one channel of the DCN system and delivers a measurement of the Faraday rotation angle with a typical noise level of $+/-0.1^{\circ}$ in stationary phases of the plasma discharge. Spurious ellipticity is present in the system and currently prevents an unbiased measurement of the Cotton-Mouton effect. The polarimeter will undergo further optimization in the near future and is likely to be extended also to the other interferometer chords.

\section{ACKNOWLEDGMENTS}

The work leading to this article was funded by the European Atomic Energy Community and is subject to the provisions of the European Fusion Development Agreement.

${ }^{1}$ A. Mlynek, G. Schramm, H. Eixenberger et al., Rev.Sci.Inst. 81, 033507 (2010).

${ }^{2}$ H. R. Koslowski, Meas. Sci. Technol. 5, 307 (1994).

${ }^{3}$ E.E. Russell, E.E. Bell, Journal of the Optical Society of America 57, 341-348 (1967).

${ }^{4}$ B. Bieg, Y. Kravtstov, M. Cieplik, Fusion Eng. Des. 88, 1452-1454 (2013). 